\title{
Editorial
}

\section{Polymyxin B Hemoperfusion in Sepsis: Growing Body of Evidence and Occasional Conflicting Results}

\author{
Massimo Antonelli ${ }^{a}$ Claudio Ronco ${ }^{b}$ \\ ${ }^{a}$ Department of Anesthesiology and Intensive Care Medicine, Catholic University of Rome, A. Gemelli University \\ Hospital, Rome, ${ }^{b}$ Department of Nephrology, Dialysis and Transplantation, International Renal Research Institute of \\ Vicenza (IRRIV), San Bortolo Hospital, Vicenza, Italy
}

Polymixin B hemoperfusion (PMX-HP) is a relatively new therapeutic strategy used in the treatment of sepsis and septic shock [1-5]. While the treatment was first initiated in Japan, it rapidly gained acceptance in Europe and is now expanding to North America. In fact, PMX$\mathrm{HP}$ is currently the subject of an ongoing North-American randomized controlled trial (EUPHRATES) in patients with septic shock and confirmed endotoxemia (measured by the endotoxin activity assay, EAA). The mechanism of action of this therapy is the removal of circulating endotoxin. High affinity binding of circulating endotoxin by the PMX-HP column may reduce circulating endotoxin levels by up to $90 \%$ after two standard treatments $(2 \mathrm{~h} \mathrm{ev}$ ery $24 \mathrm{~h}$ ). Basic science research has shown reductions in circulating cytokine levels and in renal tubular apoptosis. Clinical research has demonstrated hemodynamic improvement, better oxygenation, improved renal function, and reduction in mortality [5]. Although further research will be required to define additional patient populations with endotoxemia that may benefit from PMX-HP therapy, so far this has been considered one of the interesting options for sepsis patients where pharmacological trials have mostly been disappointing. A certain body of evidence has been accumulated so far suggesting clinical benefits in terms of short- and long-term outcomes with
(C) 2015 S. Karger AG, Basel

0253-5068/15/0393-000I\$39.50/0 special focus on recovery of organ function. Nevertheless, some conflicting results are occasionally reported in the literature and this is the case with the ABDO-MIX trial, recently published by Payen et al. [6]. In this study, the early use of polymyxin B hemoperfusion (PMX-HP) in peritonitis-induced septic shock after surgery did not induce any significant benefit in terms of outcome. The authors should be commended for their effort but with this additional piece of information, we feel that we be cautious in drawing definitive conclusions. The observed 28day mortality rate was $27.7 \%$ in the PMX-treated group and $19.5 \%$ in the control group, without any significant difference.

However, we may speculate that the 28-day mortality rate recorded for both groups was significantly lower than that reported in larger studies (between 32.7 and 53\% for similar patient cohorts) [7-9].

The initial ABDO-MIX enrolment was not achieved. Furthermore, only 81 of the 119 treated patients completed the two scheduled sessions of PMX-HP. In the previously published EUPHAS study [5], whose results were used for the ABDO-MIX sample size calculation, all patients enrolled had completed the two planned sessions of PMX-HP with a definitely higher mortality in the control group. Thus, major differences among trials jeopardize
Prof. Claudio Ronco, MD

Department of Nephrology, Dialysis and Transplantation International Renal Research Institute of Vicenza (IRRIV) San Bortolo Hospital, IT-36100 Vicenza (Italy)

E-Mail cronco@goldnet.it 
their comparability suggesting that any definitive conclusion be put on hold.

Among the reasons that have led to the noncompletion of the two PMX-HP planned, the authors indicated cartridge clotting and severe hemodynamic instability. If one considers the difference in the heparin infusion rate prescribed between the two studies $(2,000-4,000 \mathrm{UI}$ in ABDO-MIX vs. 3,000 UI bolus $+20 \mathrm{UI} / \mathrm{kg} / \mathrm{h}$ during the PMX-HP), it can be concluded that a suboptimal anticoagulation regime for the extracorporeal technique could be responsible for the higher clotting rate observed in ABDO-MIX compared to EUPHAS (11 vs. $6 \%$, respectively). Any specific contraindication in the case of haemodynamic instability or clinical complications related to hypotension, which in turn is related to extracorporeal hemoperfusion, has never been described. In particular, since fluid balance has been achieved due to zero ultrafiltration setting, there is no plausible reason for hemoperfusion-induced hemodynamic instability.

In nonresponsive septic shock, the high mortality risk matches the exclusion criteria of 'Moribund status or life expectancy lower than 48 h'. Patients affected by nonresponsive septic shock should not be excluded from the cohort of patients studied.

In ABDO-MIX, the authors only showed the distribution of microbiological findings in surgical samples because a better patients' stratification could be useful to know the mono- or poly-microbial nature of the infec- tions as well as the findings of microbiological samples other than those from the abdominal fluid. In the ABDOMIX study, isolation of yeasts from peritoneum was higher in PMX-treated group than control even though without a statistical difference. It is worth noting that a peritoneal candidosis is a severe life-threatening condition influencing the outcome and not susceptible to improve by the endotoxin-adsorption blood purification techniques. As per the IL- 6 tested in the ABDO-MIX, it is important to remind that the IL- 6 is a marker that is not specific for the infections and of poor utility to evaluate PMX-HP efficacy when infection, surgical trauma, chemical peritoneal inflammation from bile or gastrointestinal contents are inseparable conditions in the same patients.

In conclusion, conflicting results in the literature do not allow for a definitive take-home message. The ABDO-MIX study is less than conclusive and it cannot be considered a definitive answer to the original research question. We recommend waiting the results of the North-American double blind randomized control trial named EUPHRATES and the European follow-up study called EUPHAS-2. The uncertainty of results suggest caution and a careful planning of the selection of patients in future trials, a well-defined treatment prescription, an accurate and effective treatment delivery to avoid confounders and allow for a clear and solid evidence to be built from rigorously collected results [10].

\section{References}

1 Ronco C: The place of early haemoperfusion with polymyxin B fibre column in the treatment of sepsis. Crit Care 2005;9:631633.

2 Berto P, Ronco C, Cruz D, Melotti RM, Antonelli M: Cost-effectiveness analysis of polymyxin-B immobilized fiber column and conventional medical therapy in the management of abdominal septic shock in Italy. Blood $\mathrm{Pu}-$ rif 2011;32:331-340.

3 Cruz DN, Perazella MA, Bellomo R, de Cal M, Polanco N, Corradi V, Lentini P, Nalesso F, Ueno T, Ranieri VM, Ronco C: Effectiveness of polymyxin B-immobilized fiber column in sepsis: a systematic review. Crit Care 2007; 11:R47.

4 Cruz DN, Bellomo R, Ronco C: Clinical effects of polymyxin B-immobilized fiber column in septic patients. Contrib Nephrol 2007; 156:444-451.

5 Cruz DN, Antonelli M, Fumagalli R, Foltran F, Brienza N, Donati A, Malcangi V, Petrini F, Volta G, Bobbio Pallavicini FM, Rottoli F,
Giunta F, Ronco C: Early use of polymyxin B hemoperfusion in abdominal septic shock: the EUPHAS randomized controlled trial. JAMA 2009;301:2445-2452.

6 Payen DM, Guilhot J, Launey Y, Lukaszewicz AC, Kaaki M, Veber B, Pottecher J, JoannesBoyau O, Martin-Lefevre L, Jabaudon M, Mimoz O, Coudroy R, Ferrandière M, Kipnis E, Vela C, Chevallier S, Mallat J, Robert R; ABDOMIX Group: Early use of polymyxin $B$ hemoperfusion in patients with septic shock due to peritonitis: a multicenter randomized control trial. Intensive Care Med 2015, DOI: 10.1007/s00134-015-3751-z.

7 Antonelli M, Fumagalli R, Cruz DN, Brienza N, Giunta F; EUPHAS Study Group: PMX endotoxin removal in the clinical practice: results from the EUPHAS trial. Contrib Nephrol 2010;167:83-90.

8 Cruz DN, de Cal M, Piccinni P, Ronco C: Polymyxin-B hemoperfusion and endotoxin removal: lessons from a review of the literature. Contrib Nephrol 2010;167:77-82.
9 Antonelli M, Bello G, Maviglia R, Cutuli SL, Ronco C, Cruz D, Ranieri VM, Martin EL, Fumagalli R, Monti G, Vesconi S, Casella G, Piccinni P, Debitonto M, Fasanella E, Monza G, Blasetti AG, Coletta R, D'Ambrosio M, Cinnella G, Mattei A, Piscitelli E, Centonze G, Cucurachi M, Altieri G, Del Rosso G, Polidoro M, Mani RK, Stigliano N, De Trana L, Pittella G, Paternoster G, Caione R, Puscio D, Singh O, Pulito G, Idra AS, Sathe P, Congolani D, Falzetti G, Vecchiarelli P, Giunta F, Forfori F, Castiglione G, Nangia V, Madonna R, Urbano C, Pezza B, Sachin G, D'Costa PM, Capra C, Crema L, Tamayo L, Srivanas S, Singh YP; Early Use of Polymyxin B Hemoperfusion in the Abdominal Sepsis 2 Collaborative Group: Polymyxin B hemoperfusion in clinical practice: the picture from an unbound collaborative registry. Blood Purif 2014;37(suppl 1):22-25.

10 Ronco C, Klein DJ: Polymyxin B hemoperfusion: a mechanistic perspective. Crit Care 2014;18:309. 